\title{
Toward Astrophysical Turbulence in the Laboratory
}

\author{
G. G. Howes, ${ }^{1}$ D. J. Drake, ${ }^{1,2}$ K. D. Nielson, ${ }^{1}$ T. A. Carter, ${ }^{3}$ C. A. Kletzing, ${ }^{1}$ and F. Skiff ${ }^{1}$ \\ ${ }^{1}$ Department of Physics and Astronomy, University of Iowa, Iowa City, Iowa 52242, USA \\ ${ }^{2}$ Department of Physics, Astronomy, and Geosciences, Valdosta State University, Valdosta, Georgia 31698, USA \\ ${ }^{3}$ Department of Physics and Astronomy, University of California, Los Angeles, California 90095-1547, USA
}

(Received 20 July 2012; published 17 December 2012)

\begin{abstract}
Turbulence is a ubiquitous phenomenon in space and astrophysical plasmas, driving a cascade of energy from large to small scales and strongly influencing the plasma heating resulting from the dissipation of the turbulence. Modern theories of plasma turbulence are based on the fundamental concept that the turbulent cascade of energy is caused by the nonlinear interaction between counterpropagating Alfvén waves, yet this interaction has never been observationally or experimentally verified. We present here the first experimental measurement in a laboratory plasma of the nonlinear interaction between counterpropagating Alfvén waves, the fundamental building block of astrophysical plasma turbulence. This measurement establishes a firm basis for the application of theoretical ideas developed in idealized models to turbulence in realistic space and astrophysical plasma systems.
\end{abstract}

DOI: 10.1103/PhysRevLett.109.255001

PACS numbers: 52.72.+v, 52.35.Bj, 52.35.Mw, 52.35.Ra

Introduction.-Turbulence profoundly affects many space and astrophysical plasma environments, playing a crucial role in the heating of the solar corona and acceleration of the solar wind [1], the dynamics of the interstellar medium [2-4], the regulation of star formation [5], the transport of heat in galaxy clusters [6], and the transport of mass and energy into Earth's magnetosphere [7]. At the large length scales and low frequencies characteristic of the turbulence in these systems, the turbulent motions are governed by the physics of Alfvén waves [8], traveling disturbances of the plasma and magnetic field. Theories of Alfvénic turbulence based on idealized models, such as incompressible magnetohydrodynamics (MHD), suggest that the turbulent cascade of energy from large to small scales is driven by the nonlinear interaction between counterpropagating Alfvén waves [9-12]. However, the applicability of this key concept in the moderately to weakly collisional conditions relevant to astrophysical plasmas has not previously been observationally or experimentally demonstrated. Verification is important because the distinction between the two leading theories for strong MHD turbulence [11,12] arises from the detailed nature of this nonlinear interaction. Furthermore, verification is required to establish the applicability of turbulence theories, utilizing simplified fluid models such as incompressible MHD, to the weakly collisional conditions of diffuse astrophysical plasmas.

Several reasons make it unlikely that the nonlinear interaction between counterpropagating Alfvén waves can ever be verified using observations of turbulence in astrophysical environments: the spatial resolution achievable in astrophysical observations is insufficient, in situ spacecraft measurements yield information at only a single or a few spatial points, and the broad spectrum of turbulent modes confounds attempts to identify the transfer of energy from two nonlinearly interacting Alfvén waves to a third wave. Only experimental measurements in the laboratory can achieve the controlled conditions and high spatial resolution necessary. The unique capabilities of the Large Plasma Device (LAPD) at UCLA [13], designed to study fundamental plasma physics processes, make possible the first laboratory measurement of the nonlinear wave-wave interaction underlying Alfvénic turbulence.

This Letter presents the first laboratory measurement of the nonlinear interaction between counterpropagating Alfvén waves, the fundamental building block of astrophysical plasma turbulence. The properties of the nonlinear daugther Alfvén wave are predicted from incompressible MHD theory. The experimental setup and procedure are outlined. Analysis of the experimental results demonstrate a successful measurement of the nonlinear interaction between counterpropagating Alfvén waves.

Theory.-Modern theories of anisotropic Alfvénic plasma turbulence are based on several key concepts derived from the equations of incompressible MHD. These equations can be expressed in the symmetric form [14],

$$
\partial \mathbf{z}^{ \pm} / \partial t \mp\left(\mathbf{v}_{A} \cdot \nabla\right) \mathbf{z}^{ \pm}=-\left(\mathbf{z}^{\mp} \cdot \nabla\right) \mathbf{z}^{ \pm}-\nabla p / \rho_{0},
$$

where the magnetic field is decomposed into $\mathbf{B}=\mathbf{B}_{0}+$ $\delta \mathbf{B}, \mathbf{v}_{A}=\mathbf{B}_{0} / \sqrt{\mu_{0} \rho_{0}}$ is the Alfvén velocity due to the equilibrium field $\mathbf{B}_{0}, p$ is total pressure (thermal plus magnetic), $\rho_{0}$ is mass density, and $\mathbf{z}^{ \pm}=\mathbf{v}_{\perp} \pm \delta \mathbf{B}_{\perp} /$ $\sqrt{\mu_{0} \rho_{0}}$ are the Elsässer fields of the Alfvén waves which are incompressible, so that $\nabla \cdot \mathbf{z}^{ \pm}=0$. The Elsässer field $\mathbf{z}^{+}\left(\mathbf{z}^{-}\right)$represents an Alfvén wave traveling down (up) the mean magnetic field. The second term on the left-hand side of (1) is the linear term representing the propagation of the Elsässer fields along the mean magnetic field at the Alfvén speed, the first term on the right-hand side is the nonlinear 
term representing the interaction between counterpropagating waves, and the second term on the right-hand side ensures incompressibility [11].

Consider the nonlinear interaction between two plane Alfvén waves with wave vectors $\mathbf{k}_{1}$ and $\mathbf{k}_{2}$, each with nonzero components both parallel and perpendicular to the equilibrium magnetic field. The mathematical form of the nonlinear term in (1) requires two conditions for nonlinear interaction to occur: (a) both $\mathbf{z}^{+} \neq 0$ and $\mathbf{z}^{-} \neq 0$, so the two waves must propagate in opposite directions along the magnetic field, implying $k_{\| 1}$ and $k_{\| 2}$ have opposite signs [9,15]; and, (b) the polarizations of the perpendicular wave magnetic fields are not parallel, implying $\mathbf{k}_{\perp 1} \times$ $\mathbf{k}_{\perp 2} \neq 0$. These properties dictate that the fundamental building block of plasma turbulence is the nonlinear interaction between perpendicularly polarized, counterpropagating Alfvén waves.

For sufficiently small amplitudes, the terms on the righthand side of (1) are small compared to the linear propagation term, producing a state of weak MHD turbulence [10]. Note that the linear term in (1) has no counterpart in incompressible hydrodynamics, eliminating the possibility of weak turbulence, a fundamental distinction between incompressible hydrodynamic and incompressible MHD systems. In the weak MHD turbulence paradigm, two counterpropagating Alfvén waves interact nonlinearly to transfer energy to a third wave. This is the fundamental interaction underlying the cascade of energy to small scales in plasma turbulence. Solving for the nonlinear evolution using perturbation theory demonstrates that the nonlinear three-wave interaction, averaged over many wave periods, must satisfy the constraints

$$
\mathbf{k}_{1}+\mathbf{k}_{2}=\mathbf{k}_{3} \text { and } \omega_{1}+\omega_{2}=\omega_{3} \text {, }
$$

equivalent to the conservation of momentum and energy $[10,16,17]$. Given the dispersion relation for Alfvén waves, $\omega=\left|k_{\|}\right| v_{A}$, and the requirement for counterpropagating waves, the only nontrivial solution to these equations has either $k_{\| 1}=0$ or $k_{\| 2}=0$ [18].

The frequency constraint in (2) ceases to hold when the interaction spans only a fraction of a wave period. For such brief interactions, energy is transferred nonlinearly to a daughter mode at the instantaneous rate given by the nonlinear term in (1). In the laboratory, this can be accomplished by interacting a high-frequency Alfvén wave $\mathbf{k}_{1}$ with a counterpropagating Alfvén wave $\mathbf{k}_{2}$ of much lower frequency, such that its parallel wavelength is much longer than the length over which the waves interact. The physical effect of the low-frequency wave is to generate a shear in the equilibrium magnetic field, producing an effective $k_{\| 2}=0$ component to the interaction. The predicted nonlinear product is an Alfvén wave $\mathbf{k}_{3}$ with the properties $k_{\| 3}=k_{\| 1}$ and $\mathbf{k}_{\perp 3}=\mathbf{k}_{\perp 1}+\mathbf{k}_{\perp 2}$.

Experiment.-The first experiment to verify this interaction in the laboratory was performed on the LAPD [13] using a background axial magnetic field of $800 \mathrm{G}$ to confine a hydrogen plasma in a cylindrical column of $16.5 \mathrm{~m}$ length and $40 \mathrm{~cm}$ diameter. The plasma discharge exists for approximately $11 \mathrm{~ms}$ with a repetition rate of $1 \mathrm{~Hz}$. The electron temperature, $T_{e}=5.0 \mathrm{eV}$, and density, $n=10^{12} \mathrm{~cm}^{-3}$, were determined using a swept Langmuir probe, with a microwave interferometer used to calibrate density. The ion temperature, $T_{i}=1.25 \mathrm{eV}$, was estimated from previous interferometric measurements. For these parameters, the electron-ion collision frequency [19] is $3 \mathrm{kHz}$ and the characteristic perpendicular scale is given by the ion sound Larmor radius $\rho_{s}=$ $\sqrt{T_{e} / m_{i}} / \Omega_{i}=0.29 \mathrm{~cm}$, where $\Omega_{i}$ is the ion cyclotron frequency.

The experimental setup is depicted in Fig. 1. At one end, the Arbitrary Spatial Waveform (ASW) antenna [20,21] generates an Alfvén wave (blue) with frequency $f=$ $270 \mathrm{kHz}$ and a wave magnetic field polarized in the $y$ direction, $\delta B_{y}$, characterized by a precise perpendicular wave vector $\mathbf{k}_{\perp 1} \rho_{s}=0.16 \hat{\mathbf{x}}$. On the other end, a Loop antenna [22], constructed of two crossed current loops phased to produce a dominantly horizontal wave magnetic field $\delta B_{x}$, generates a large-amplitude Alfvén wave (red) with much lower frequency $f=60 \mathrm{kHz}$, characterized by a wave vector dominated by a $y$ component $k_{\perp 2 y} \rho_{s}=$ 0.055 . The perpendicular wave magnetic fields, $\delta B_{x}$ and $\delta B_{y}$, are measured using an Elsässer probe [23] between the antennas approximately $2 \mathrm{~m}$ from the Loop antenna and $9 \mathrm{~m}$ from the ASW antenna. The measurements are taken over a perpendicular plane of size $30 \mathrm{~cm} \times 30 \mathrm{~cm}$ at a spacing of $0.75 \mathrm{~cm}$, yielding a spatial grid of $41 \times 41$ measurement positions. At each position, 10 shots are averaged to improve the signal-to-noise ratio.

To generate a nonlinear interaction between the counterpropagating Loop and ASW Alfvén waves, first the Loop antenna is turned on and allowed us to establish a steady wave pattern at $f=60 \mathrm{kHz}$. Next, the ASW antenna

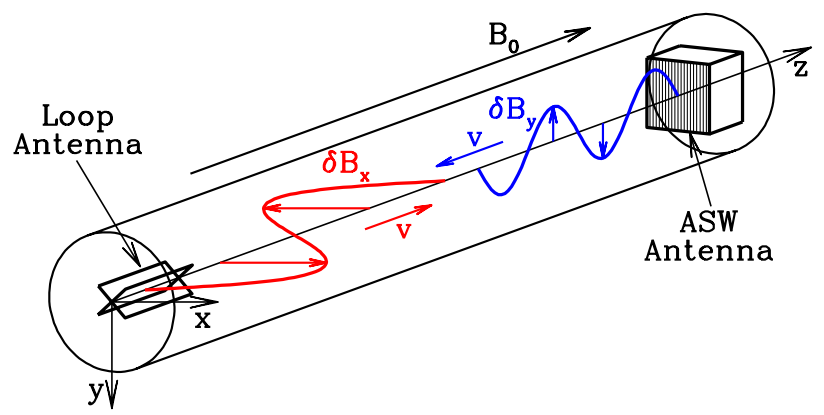

FIG. 1 (color online). Schematic of the Alfvén wave turbulence experiment on the LAPD. The Loop antenna generates a large-amplitude Alfvén wave polarized in the $x$ direction traveling up the mean magnetic field $B_{0}$ and the ASW antenna generates a smaller amplitude Alfvén wave polarized in the $y$ direction traveling down the mean magnetic field. 

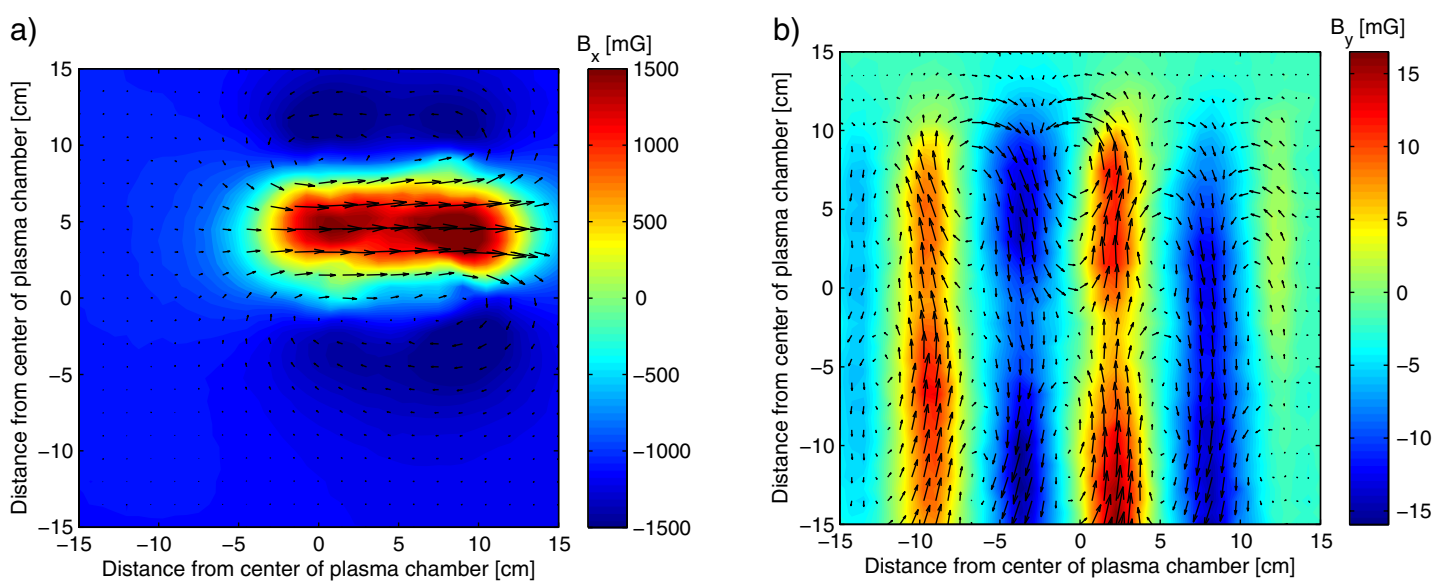

FIG. 2 (color online). Spatial magnetic-field waveforms from the (a) Loop antenna and (b) ASW antenna. The color map indicates (a) $\delta B_{x}$ for the Loop antenna and (b) $\delta B_{y}$ for the ASW antenna in $\mathrm{mG}$, and arrows indicate the vector direction of $\delta \mathbf{B}_{\perp}$.
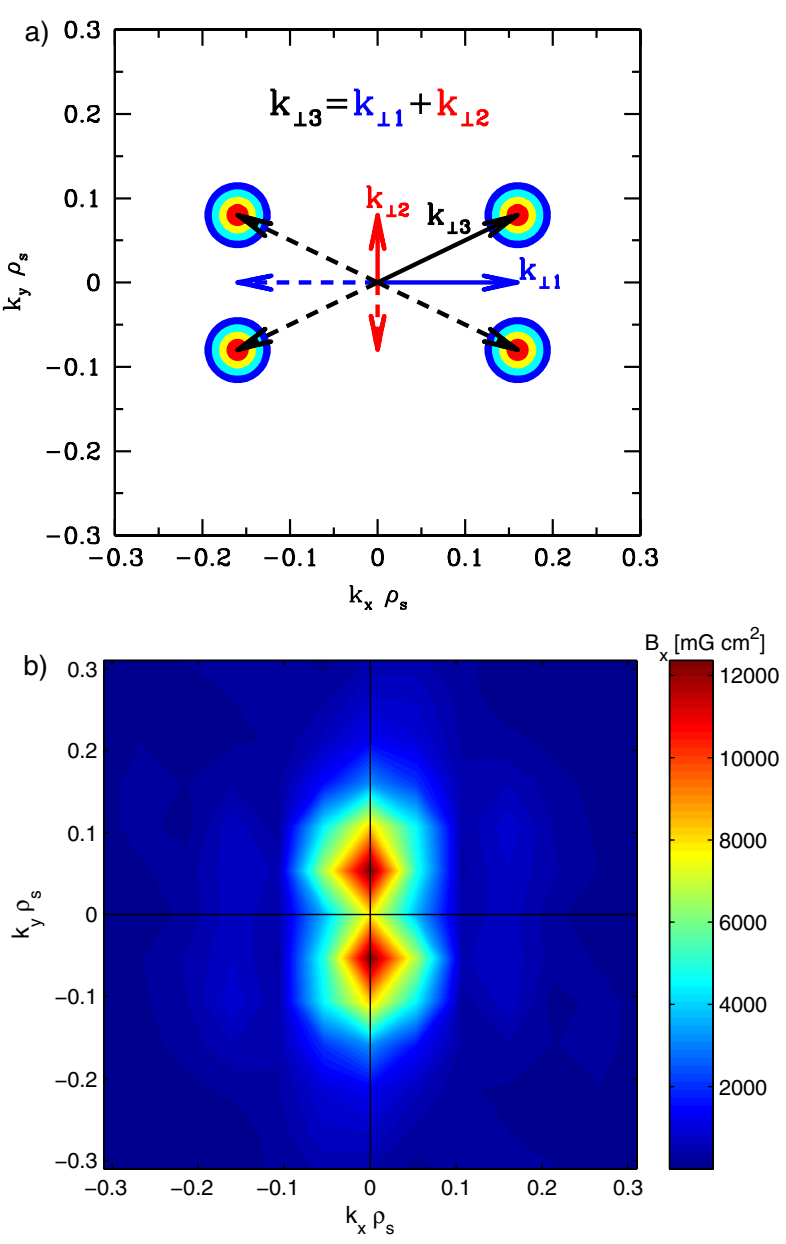
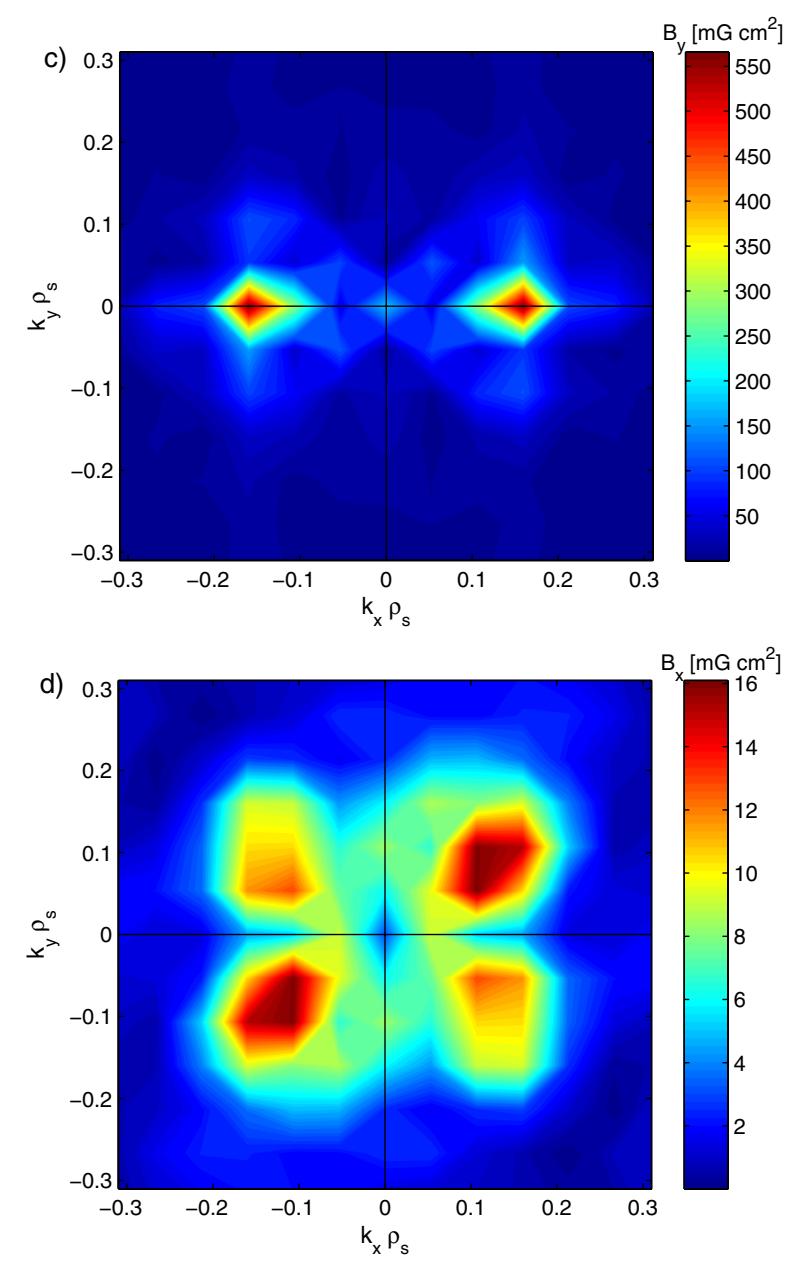

FIG. 3 (color online). (a) Diagram of $\mathbf{k}_{\perp}$ for the ASW antenna (blue) and Loop antenna (red). For the nonlinear daughter Alfvén wave, $\mathbf{k}_{\perp 3}$ (black) is the vector sum of the two antenna wave vectors, $\mathbf{k}_{\perp 3}=+\mathbf{k}_{\perp 1} \pm \mathbf{k}_{\perp 2}$ and $\mathbf{k}_{\perp 3}=-\mathbf{k}_{\perp 1} \pm \mathbf{k}_{\perp 2}$. Bullseyes indicate predicted power distribution of the nonlinear product. (b) Color map of $\delta B_{x}\left(k_{x}, k_{y}\right)$ for the Loop antenna by itself. (c) Color map $\left(\mathrm{mG} \mathrm{cm}^{2}\right)$ of $\delta B_{y}\left(k_{x}, k_{y}\right)$ for the ASW antenna by itself. (d) Color map of $\delta B_{x}\left(k_{x}, k_{y}\right)$ for the nonlinear daughter Alfvén wave. 
launches a perpendicularly polarized, counterpropagating wave. The spatial antenna waveform $\delta \mathbf{B}_{\perp}$, measured in single-antenna runs with identical timing, is presented in Fig. 2. Color maps show the magnitude of (a) $\delta B_{x}$ for the Loop antenna and (b) $\delta B_{y}$ for the ASW antenna, and arrows show the vector direction of $\delta \mathbf{B}_{\perp}$. The ASW antenna produces a waveform of $30 \mathrm{mG}$ peak-to-peak amplitude with little $\delta B_{x}$ component, whereas the Loop antenna produces a waveform of $3000 \mathrm{mG}$ peak-to-peak amplitude that is dominated by the $\delta B_{x}$ component.

The experimental results are presented in Fig. 3. We plot (b) the Fourier transform in the perpendicular plane, $\delta B_{x}\left(k_{x}, k_{y}, t\right)$, for the Loop antenna by itself and (c) $\delta B_{y}\left(k_{x}, k_{y}, t\right)$ for the ASW antenna by itself. Note that these are the spatial Fourier transforms of the antenna patterns in Fig. 2, and both antennas generate a pair of waves with $\pm \mathbf{k}_{\perp}$.

The analysis of the counterpropagating Alfvén wave run is designed to identify the nonlinear daughter Alfvén wave with two distinguishing properties: (a) $k_{\| 3}=k_{\| 1}$, which means the daughter wave will have the same frequency as the ASW wave, and (b) $\mathbf{k}_{\perp 3}=\mathbf{k}_{\perp 1}+\mathbf{k}_{\perp 2}$. First, to remove the linear contribution to $\delta B_{x}$ caused by the large-amplitude Loop Alfvén wave, we subtract the signal of $\delta B_{x}$ of the Loop-antenna-only run from the corresponding signal of the counterpropagating run to obtain $\delta B_{x}(x, y, t)$. Next, we Fourier transform in time the interval with both waves to obtain $\delta B_{x}(x, y, f)$. Since the signal of the daughter wave is expected to peak at the same frequency $f=270 \mathrm{kHz}$ as the ASW wave, we bandpass filter the $\delta B_{x}(x, y, f)$ signal over the frequency range $170 \mathrm{kHz} \leq f \leq 370 \mathrm{kHz}$, setting the Fourier coefficients to zero outside this range. The filtered frequency signal is then inverse Fourier transformed from frequency back to time. Finally, we Fourier transform in the perpendicular plane to obtain the spatial Fourier transform of the daughter wave, $\delta B_{x}\left(k_{x}, k_{y}, t\right)$.

The result of this procedure, shown in panel (d) of Fig. 3, is the key experimental result of this Letter. The observational signature of the nonlinear daughter Alfvén wave is clear, with the wave field $\delta B_{x}$ dominated by four wave vectors, corresponding to all possible sums of perpendicular antenna wave vectors, $\mathbf{k}_{\perp 3}=+\mathbf{k}_{\perp 1} \pm \mathbf{k}_{\perp 2}$ and $\mathbf{k}_{\perp 3}=-\mathbf{k}_{\perp 1} \pm \mathbf{k}_{\perp 2}$, as shown schematically in panel (a) of Fig. 3. The noise level of the subtracted signal in Fourier space is $1.2 \mathrm{mG} \mathrm{cm}^{2}$, yielding a signal to noise ratio $S / N \geqslant 10$, demonstrating that the measurement is physically meaningful. In addition, the amplitude of this nonlinear daughter Alfvén wave, which agrees to order of magnitude with theoretical expectations, peaks at the ASW wave frequency, $f_{3}=270 \mathrm{kHz}$, as predicted. These results demonstrate that we have successfully measured the nonlinear interaction between counterpropagating Alfvén waves, the fundamental building block of astrophysical plasma turbulence.
This experimental finding verifies that the general properties of the nonlinear interaction between counterpropagating Alfvén waves, as derived theoretically in the idealized context of incompressible MHD, hold even under the weakly collisional conditions relevant to many space and astrophysical plasma environments. Although these conditions formally require a kinetic description of the turbulent dynamics, our results indicate that the key concepts derived from reduced fluid models describe the essential nature of the turbulent interactions. Future experiments will probe the turbulent dynamics at smaller scales, providing guidance for the extension of existing turbulence theories into the uncharted regime of kinetic turbulence at scales below the ion sound Larmor radius, where the effects of wave dispersion and kinetic wave-particle interactions influence the turbulent dynamics.

Supported by NSF Grants No. ATM 03-17310 and No. PHY-10033446, DOE Grant No. DE-FG0206ER54890, NSF CAREER Award AGS-1054061, and NASA Grant No. NNX10AC91G. The experiment presented here was conducted at the Basic Plasma Science Facility, funded by the U. S. Department of Energy and the National Science Foundation.

[1] S. W. McIntosh, B. de Pontieu, M. Carlsson, V. Hansteen, P. Boerner, and M. Goossens, Nature (London) 475, 477 (2011).

[2] J. W. Armstrong, J. M. Cordes, and B. J. Rickett, Nature (London) 291, 561 (1981).

[3] J. W. Armstrong, B.J. Rickett, and S.R. Spangler, Astrophys. J. 443, 209 (1995).

[4] B. M. Gaensler, M. Haverkorn, B. Burkhart, K. J. NewtonMcGee, R. D. Ekers, A. Lazarian, N. M. McClureGriffiths, T. Robishaw, J.M. Dickey, and A.J. Green, Nature (London) 478, 214 (2011).

[5] B. J. Rickett, Annu. Rev. Astron. Astrophys. 28, 561 (1990).

[6] J. R. Peterson and A. C. Fabian, Phys. Rep. 427, 1 (2006).

[7] D. Sundkvist, V. Krasnoselskikh, P. K. Shukla, A. Vaivads, M. André, S. Buchert, and H. Rème, Nature (London) 436, 825 (2005).

[8] H. Alfvén, Nature (London) 150, 405 (1942).

[9] R. H. Kraichnan, Phys. Fluids 8, 1385 (1965).

[10] S. Sridhar and P. Goldreich, Astrophys. J. 432, 612 (1994).

[11] P. Goldreich and S. Sridhar, Astrophys. J. 438, 763 (1995).

[12] S. Boldyrev, Phys. Rev. Lett. 96, 115002 (2006).

[13] W. Gekelman, H. Pfister, Z. Lucky, J. Bamber, D. Leneman, and J. Maggs, Rev. Sci. Instrum. 62, 2875 (1991).

[14] W. M. Elsasser, Phys. Rev. 79, 183 (1950).

[15] R. S. Iroshnikov, Astron. Zh. 40, 742 (1963) [Sov. Astron. 7566 (1964)].

[16] C.S. Ng and A. Bhattacharjee, Astrophys. J. 465, 845 (1996).

[17] S. Galtier, S. V. Nazarenko, A. C. Newell, and A. Pouquet, J. Plasma Phys. 63, 447 (2000). 
[18] J. V. Shebalin, W.H. Matthaeus, and D. Montgomery, J. Plasma Phys. 29, 525 (1983).

[19] S. I. Braginskii, Rev. Plasma Phys. 1, 205 (1965).

[20] D. J. Thuecks, C. A. Kletzing, F. Skiff, S. R. Bounds, and S. Vincena, Phys. Plasmas 16, 052110 (2009).
[21] C. A. Kletzing, D. J. Thuecks, F. Skiff, S. R. Bounds, and S. Vincena, Phys. Rev. Lett. 104, 095001 (2010).

[22] D. W. Auerbach, T. A. Carter, S. Vincena, and P. Popovich, Phys. Plasmas 18, 055708 (2011).

[23] D. J. Drake, C. A. Kletzing, F. Skiff, G. G. Howes, and S. Vincena, Rev. Sci. Instrum. 82, 103505 (2011). 